\title{
Consorcio público-público para el desarrollo de medicamentos contra la tuberculosis
}

\author{
Marianela Morzán \\ Área de Comunicación. \\ Secretaría de Vinculación \\ Tecnológica y Desarrollo Productivo. \\ Universidad Nacional del Litoral.
}

La tuberculosis es una enfermedad que afecta principalmente a los pulmones pero que puede atacar otras partes del cuerpo. Es causada por una bacteria y se contagia por el aire (cuando los enfermos tosen o estornudan). Entre los síntomas que permiten detectarla se encuentran la tos persistente, sudoración nocturna, cansancio, pérdida de peso y falta de apetito.

Si la enfermedad es detectada en forma temprana y tratada adecuadamente puede curarse. Los tratamientos duran más de seis meses, sin embargo los síntomas suelen mejorar alrededor de las tres semanas. En Argentina, alrededor de 11000 habitantes se enferman de tubersulosis cada año y entre 600 y 900 fallecen por su causa. Esta afección en general está relacionada con poblaciones que tienen amplias carencias, razón por la cual depende de planes y programas públicos para curarse.

La Universidad Nacional del Litoral (UNL), mediante sus investigadores, y el Laboratorio Industrial Farmacéutico de Santa Fe (LIF), con sus especialistas, se encuentran ejecutando un proyecto que busca optimizar y poner a punto la tecnología necesaria para la producción en la provincia de Santa Fe (Argentina) de cinco fármacos para el tratamiento de esta enfermedad.

Conforme lo señaló Carlos Arqués, director del LIF:

"La importancia de desarrollar estos medicamentos radica en que en la actualidad hay muchas dificultades para la provisión de los mismos, porque algunos no se producen en el país y otros sí pero tienen una oferta muy concentrada. Esto impacta en la adherencia del paciente a los tratamientos, lo que lleva a la aparición de tuberculosis multirresistentes, que son mucho más complejas para tratar".

Ello debido a que uno de los principales problemas es la adquisición de estos medicamentos por parte de los enfermos puesto que los principales afectados son de escasos recursos.

\section{Desarrollo del trabajo}

El proyecto se desarrolla en el marco de un consorcio público-público entre la Universidad y el laboratorio perteneciente al gobierno de la provincia de Santa Fe. Tiene la finalidad de apoyar al sistema de producción pública de medicamentos para cubrir áreas estratégicas y de vacancia en la provisión de especialidades medicinales prioritarias, articulando y complementando las capacidades del laboratorio de producción pública de medicamentos y actores del sistema científico tecnológico nacional. Fue presentado al Fondo Argentino Sectorial de la Agencia Nacional de Promoción Científica y Tecnológica, del Ministerio de Ciencia, Tecnología e Innovación Productiva de la Nación, durará tres años, y tiene un presupuesto total superior a los 39000000 de pesos, de los cuales 23373000 serán financiados por el Fondo y el resto por los integrantes del consorcio.
El trabajo será realizado por tres tipos de actores:

- Especialistas del LIF que cuentan con una vasta experiencia en el desarrollo y producción de medicamentos.

- Investigadores de la UNL que se encuentran trabajando en la generación de tecnología aplicada en estos temas.

- Gestores del Centro para la Transferencia de los Resultados de la Investigación (CETRI-Litoral, UNL) que se ocuparán de la gestión del proyecto, la administración de los fondos y el seguimiento técnico.

\section{División de tareas}

"La Universidad se involucra a partir de distintas unidades, como el Laboratorio de Control de Calidad de Medicamentos de la Facultad de Bioquímica y Ciencias Biológicas; del equipo de Caracterización de Sólidos Farmacéuticos de la Facultad de Ingeniería Química, y el CETRI Litoral, que fue el encargado de la elaboración del proyecto y es la Unidad de Vinculación Tecnológica a partir de la cual se lleva a cabo la gestión y la administración del proyecto durante estos tres años", explicó Albor Cantard, rector de la mencionada casa de estudios.

El Laboratorio de Control de Calidad de Medicamentos se ocupará de hacer todos los desarrollos necesarios para los controles de calidad de las materias primas y demás 


\section{Más información}

www.santafe.gob.ar

www.unl.edu.ar

insumos que van a intervenir en los tuberculostáticos. Asimismo, realizará actividades relacionadas con la determinación de la fórmula y tendrá la función de formar dos analistas que luego se van a incorporar a la planta del LIF para actividades de desarrollo y validación de metodologías analíticas. En lo que respecta a las actividades de desarrollo, puesta a punto y transferencia de técnicas de caracterización de las materias primas con las que se harán estos fármacos, estarán a cargo del equipo de Caracterización de Sólidos Farmacéuticos. Allí, los investigadores analizarán las materias primas, los componentes de la mezcla para formará los comprimidos, buscando tener una producción uniforme en el tiempo. Además, en ese ámbito se formarán dos analistas que se incorporarán a los equipos del LIF para esas tareas.

Por último, de las etapas vinculadas a la puesta a punto de la tecnología para la producción de los tuberculostáticos y la obtención de los lotes que serán presentados a la Administración Nacional de Medicamentos, Alimentos y Tecnología Médica de Argentina (ANMAT) para su habilitación, serán responsabilidad del LIF y este laboratorio será acompañado en su ejecución por el Departamento de Ingeniería Industrial de la Facultad de Ingeniería Química de la UNL. La ejecución del proyecto será en la sede del Laboratorio Industrial Farmacéutico Sociedad de Estado, que actualmente produce el $94 \%$ de las unidades farmacológicas que la provincia aporta para atención primaria de la salud. Sus medicamentos también se utilizan en el sistema de salud público de otras provincias argentinas y en hospitales y programas nacionales de prevención. En este marco, se instalará una planta piloto para la producción de tuberculostáticos y se pondrá a punto un laboratorio para la realización de determinaciones analíticas.
Desde el CETRI-Litoral, organismo con amplia experiencia en la formulación y gestión de proyectos de inversión, se llevarán a cabo la gestión y la administración los fondos provenientes del proyecto.

\section{Impacto social y académico}

Con este proyecto, el Consorcio se plantea el desafío de desarrollar y poner a punto a escala piloto la tecnología necesaria para luego, a partir del escalado industrial, lograr dar respuesta a estas necesidades de medicamentos para la tuberculosis en cantidades estables y suficientes y a valores razonables en función de los costos de producción, de modo de beneficiar a los sectores socialmente más desfavorecidos. En este sentido, el LIF incrementará su personal capacitado para asegurar la calidad de los productos. Esto significa también una instancia de inserción laboral para graduados de la UNL, que se formarán en el transcurso de los tres años que durará el proyecto.

A su vez, la incorporación en el laboratorio estatal de innovaciones, tecnologías y nuevas metodologías de trabajo fortalecerá las áreas de desarrollo de medicamentos, control de materias primas, preparaciones y productos relacionados y la puesta a punto del laboratorio de bioequivalencia, permitirá mejorar sustancialmente las performances de los procesos de producción de otros medicamentos elaborados por el LIF actualmente y dejará instalado un alto potencial para ampliar la cobertura de producción a otros fármacos.

La integración entre la Universidad y el laboratorio estatal en un Consorcio público-público es el resultado de una relación de más de 20 años en trabajos compartidos. De esta interacción en particular se espera obtener un enriquecimiento y aprendizaje que serán

\section{Contacto}

Secretaría de Vinculación Tecnológica

y Desarrollo Productivo.

Centro para la Transferencia

de los Resultados de la Investigación

(CETRI-Litoral) E-mail: cetri@unl.edu.ar

aprehendidos por cada uno de los miembros del Consorcio y posteriormente aprovechados en su actividad ordinaria.

El caudal de conocimientos científicos nuevos que se generarán en la UNL, así como la adquisición de equipos y la mejora de infraestructuras en el LIF, que complementa la transferencia del conocimiento desde la UNL, podrán ser volcados y transferidos en nuevos servicios y productos tanto para el LIF como para la industria en su conjunto, lo cual ayudará a potenciar las capacidades de todo el sector.

Al respecto, Mario Drisun, ministro de Salud de la provincia de Santa Fe, expresó que "esta historia trasciende varios gobiernos entre el LIF y la Universidad"; y agregó que "el encuentro entre la Universidad y el gobierno parece fundamental, especialmente a la hora de establecer políticas de Estado. Y aquí aparece otra cuestión virtuosa, que es la presencia del gobierno de la Nación a través de fondos que promueven este tipo de proyectos y que nos permiten, a la provincia y a la Universidad, encontrarnos y crecer. Estos encuentros potencian enormemente las posibilidades".

En este sentido, se espera abordar proyectos cada vez más importantes y más complejos para la generación de fármacos que curen enfermedades que afecten a la población. 